University of New Hampshire

University of New Hampshire Scholars' Repository

\title{
The Hippocampus is Preferentially Associated with Memory for Spatial Context
}

\author{
Robert S. Ross \\ The University of New Hampshire, robert.ross@unh.edu \\ Scott D. Slotnick \\ Boston College
}

Follow this and additional works at: https://scholars.unh.edu/psych_facpub

\section{Recommended Citation}

Ross, R. S., \& Slotnick, S. D. (2008). The Hippocampus is preferentially associated with memory for spatial context. Journal of Cognitive Neuroscience, 20(3), 432-446. http://doi.org/10.1162/jocn.2008.20035

This Article is brought to you for free and open access by the College of Liberal Arts (COLA) at University of New Hampshire Scholars' Repository. It has been accepted for inclusion in Psychology Scholarship by an authorized administrator of University of New Hampshire Scholars' Repository. For more information, please contact Scholarly.Communication@unh.edu. 


\title{
The Hippocampus is Preferentially Associated with Memory for Spatial Context
}

\author{
Robert S. Ross and Scott D. Slotnick
}

\begin{abstract}
The existence of a functional-anatomic dissociation for retrieving item versus contextual information within subregions of the medial temporal lobe (MTL) is currently under debate. We used a spatial source memory paradigm during eventrelated functional magnetic resonance imaging to investigate this issue. At study, abstract shapes were presented to the left or right of fixation. During test, old and new shapes were presented at fixation. Participants responded whether each shape had been previously presented on the "left," the "right," or was "new." Activity associated with contextual memory (i.e., source memory) was isolated by contrasting accurate versus inaccurate memory for spatial location. Item-memory-
\end{abstract}

\section{INTRODUCTION}

Memory retrieval has long been associated with the medial temporal lobe (MTL), which includes the hippocampus, entorhinal, perirhinal, and parahippocampal cortices (Squire, Stark, \& Clark, 2004; Eichenbaum, 2000). However, the specific roles of these MTL subregions during item and contextual memory retrieval is under debate (Gold et al., 2006; Manns, Hopkins, Reed, Kitchener, \& Squire, 2003; Yonelinas et al., 2002; Stark \& Squire, 2001). Item memory refers to memory of a previous exposure to a single item, whereas source memory refers to memory for the context in which an item was previously presented (where source memory is assumed to rely on retrieval of an item's context; Dobbins, Simons, \& Schacter, 2004; Weis et al., 2004; Donaldson \& Rugg, 1998). Event-related functional magnetic resonance imaging (fMRI) studies have shown that activity during encoding in the perirhinal cortex predicts subsequent item memory retrieval, whereas activation during encoding in the hippocampus and parahippocampal cortex predicts subsequent source memory retrieval (Davachi, Mitchell, \& Wagner, 2003; Ranganath et al., 2003), suggesting a functional distinction in MTL subregions during encoding of item and contextual information.

The hippocampus is hypothesized to be important for binding information together during memory retrieval (Eichenbaum, 2000). One theory proposes that the hippocampus binds together spatial contextual informa-

Boston College related activity was isolated by contrasting accurate item recognition without contextual memory with forgotten items. Source memory was associated with activity in the hippocampus and parahippocampal cortex. Although item memory was not associated with unique MTL activity at our original threshold, a region-of-interest (ROI) analysis revealed itemmemory-related activity in the perirhinal cortex. Furthermore, a functional-anatomic dissociation within the parietal cortex for retrieving item and contextual information was not found in any of three ROIs. These results support the hypothesis that specific subregions in the MTL are associated with item memory and memory for context.

tion processed in the parahippocampal cortex with item information processed in the perirhinal cortex (Manns \& Eichenbaum, 2006), which would suggest hippocampus and parahippocampal cortex involvement during source memory. Consistent with this view, source memory activation has been seen in both the hippocampus (Dobbins, Rice, Wagner, \& Schacter, 2003; Cansino, Maquet, Dolan, \& Rugg, 2002) and the parahippocampal cortex (Burgess, Maguire, Spiers, \& O'Keefe, 2001), suggesting that these regions play a role during contextual memory.

The perirhinal cortex is hypothesized to mediate familiarity-based item recognition (Holdstock, 2005; Brown \& Aggleton, 2001) consistent with a role during item memory. Electrophysiological recordings in both monkeys (Xiang \& Brown, 1998; Fahy, Riches, \& Brown, 1993) and humans (Halgren et al., 2006) have shown that neurons in the perirhinal cortex decrease their firing rate upon subsequent exposure to a stimulus. It has also been shown that perirhinal cortex activity decreases during retrieval of item information without context compared to correct rejections and retrieval of item information with context in humans using fMRI (Weis et al., 2004). Other event-related fMRI studies using words and scenes have also suggested that decreased activity in the perirhinal cortex signals familiarity (Montaldi, Spencer, Roberts, \& Mayes, 2006; Henson, Cansino, Herron, Robb, \& Rugg, 2003), suggesting perirhinal cortical involvement in item memory.

Outside the MTL, regions of the parietal cortex have shown functional differences when participants perform 
the remember/know task. A region in the intraparietal sulcus near Brodmann's area (BA) 40/39 has been shown to increase activity when retrieving old information compared to correct rejections (Wheeler \& Buckner, 2003, 2004; Konishi, Wheeler, Donaldson, \& Buckner, 2000). In a study using the remember/know procedure, where participants were instructed to respond "remember" when previously studied items are accompanied by awareness of specific aspects of the stimuli's occurrence or "know" when previously studied items are accompanied by a feeling of familiarity, Wheeler and Buckner (2004) showed that regions lateral and posterior to the BA 40/39 region just described are associated with greater activity during remember responses compared to both correct rejections and know responses. It is noteworthy that these remember-related "increases" in lateral and posterior parietal regions were driven by deactivations (relative to the baseline level of activity) in response to both correct rejections and know responses.

Our primary aim in the present event-related fMRI study was to determine whether item and source memory are associated with differential processing within specific subregions of the MTL (i.e., the hippocampus, parahippocampal cortex, and perirhinal cortex). Our secondary aim was to characterize item and context memory related activity in the parietal cortex regions of interest (ROIs) described above. In the study phase of our paradigm, abstract shapes were shown to the left and right of fixation, whereas the test phase consisted of old and new shapes presented at fixation. At test, participants responded whether each shape was old and on the "left," old and on the "right," or was "new" (Figure 1). One advantage of using abstract shapes as stimuli is that item memory and source memory accuracy did not differ, as it has in previous studies, suggesting that the present results do not suffer from a task difficulty confound. Of relevance to our primary aim, spatial source-memory-related increases in the hippocampus and parahippocampal cortex should be observed if these regions are associated with preferential processing of contextual memory. Furthermore, item memory for abstract shapes should be associated with an activation decrease in the perirhinal cortex if the perirhinal cortex does indeed process item familiarity by a decrease in neural activity. To anticipate the results, the expected pattern of activity was observed supporting the hypothesis that specific subregions of the MTL are associated with item memory and memory for context.

\section{METHODS}

\section{Participants}

Sixteen right-handed, native English-speaking participants between the ages of 18 and 35 years with normal or corrected-to-normal vision were each paid $\$ 100$ to take part in the study. Four participants did not com-

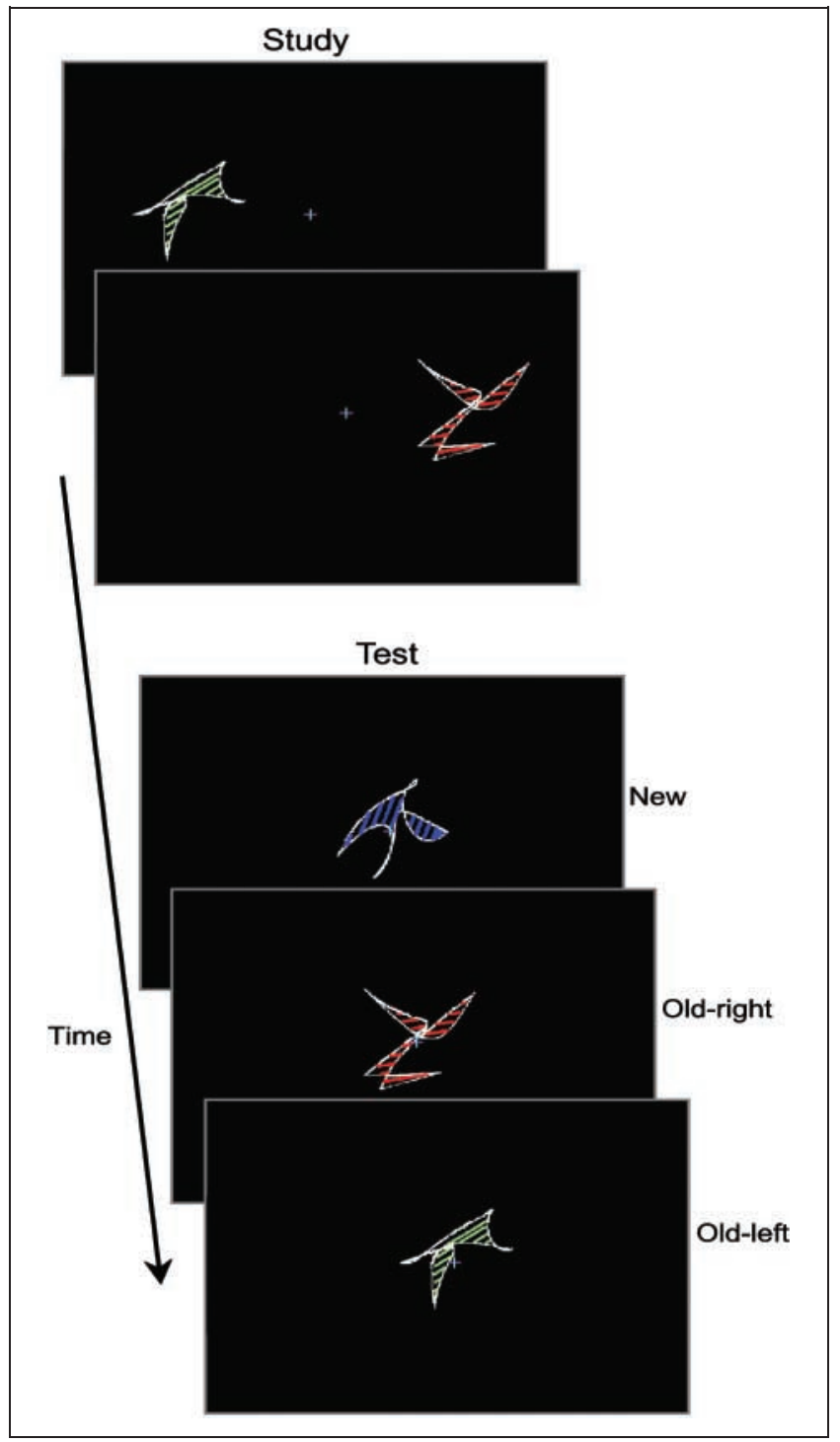

Figure 1. Behavioral protocol. During the study phase, shapes were presented to the left or right of fixation. During the test phase, shapes that had been previously presented on the left, shapes that had been previously presented on the right, and new shapes were presented at fixation (correct responses are shown to the right of each shape).

plete the full complement of study-test runs and were eliminated from the study, restricting the analysis to 12 participants with an average age of $21 \pm 0.75$ (mean \pm $S E M)$ years (7 women, 5 men). The experimental protocol was approved by the Massachusetts General Hospital Internal Review Board and informed consent was obtained from each participant.

\section{Experimental Protocol}

All participants completed one study-test run for training purposes prior to scanning followed by six study-test runs during fMRI. In each study phase, 32 unrelated shapes were sequentially presented every $3 \mathrm{sec}(2.5 \mathrm{sec}$ duration), half of the shapes were presented at $3^{\circ}$ 
visual angle to the left of fixation, whereas the other half were presented at $3^{\circ}$ visual angle to the right of fixation using a pseudorandom assignment of spatial location (no more than four items were sequentially presented on a given side). Shapes were generated in MATLAB (The MathWorks Inc.) using custom software written by S.D.S. and were composed of four pseudorandomly generated Bezier curves, each with end points on adjacent sides of a bounding square with an edge length of $5.5^{\circ}$ visual angle (for additional shape construction details, see Slotnick \& Schacter, 2004). Each shape was filled with lines of a pseudorandomly generated color and orientation (Figure 1).

Participants were instructed to remember each shape and its spatial location. During each test phase, participants were sequentially shown each of the 32 shapes from the study phase in addition to 16 new shapes at fixation in a pseudorandom order every 4-12 sec (8 sec average, duration $2.5 \mathrm{sec}$; no more than three items in a given condition were sequentially presented). Shapes were never repeated across runs (except for old shapes in the test phase), whereas line color and orientation were repeated across runs, but never within a run (except for studied/old shapes in the test phase). Participants responded to each stimulus during the test phase by pressing: (1) if they thought the stimulus was old and had previously been presented on the "left," (2) if they thought the stimulus was "new," and (3) if they thought the stimulus was old and had previously been presented on the "right" (i.e., a recognition/source memory judgment). Participants were instructed that both response speed and accuracy were equally important. Old-left, old-right, and new lists of shapes were counterbalanced across participants using a Latin square design.

\section{Imaging Acquisition and Preprocessing}

Imaging was conducted using a 3-T Siemens Allegra scanner with a standard head coil. Anatomic images were acquired using a multiplanar rapidly acquired gradientecho (MP-RAGE) sequence $(\mathrm{TR}=30 \mathrm{sec}, \mathrm{TE}=3.3 \mathrm{msec}$, slices $=128$, resolution $=1 \mathrm{~mm} \times 1 \mathrm{~mm} \times 1.33 \mathrm{~mm}$ ). Functional images were acquired using an echo-planar imaging (EPI) sequence ( $\mathrm{TR}=2 \mathrm{sec}, \mathrm{TE}=30 \mathrm{msec}$, acquisition matrix $=64 \times 64$, slices $=30$, resolution $=$ $4.5 \mathrm{~mm}$ isotropic). Imaging analysis was conducted using SPM5 (Wellcome Department of Cognitive Neurology, London, UK). Images were slice-time corrected, motion corrected, and spatially normalized to the Montreal Neurological Institute (MNI) template with voxel resampling at $2 \mathrm{~mm}$ isotropic resolution. To maximize spatial accuracy of the results, no spatial smoothing was applied.

\section{Data Analysis}

Analysis was conducted using a general linear model approach. On an individual participant basis, a canonical hemodynamic response function was convolved with a series of square waves defined by each event onset and the subsequent behavioral response to produce that event's hemodynamic response model. On an individual voxel basis, all event hemodynamic response models were fit to the voxel's activation time course using a general linear model to obtain the best-fit event model amplitudes (i.e., beta-weights). Voxels were deemed active for each statistical contrast of interest when the difference between the associated beta-weights was significantly positive using a one-tailed $t$ test, where variance was estimated using between-participant variability (i.e., a random effect analysis). One-tailed $t$ tests were employed because the magnitude of beta-weight differences in the contrasts of interest was determined a priori, based on cognitive analysis and previous results (all $t$ values are therefore positive and indicate an increase in activation for the contrast of interest). Each event's beta-weight reflected the degree to which that event reflected activity within a given voxel.

Events during encoding were sorted based on each participant's responses during the test phase. Events included successful encoding of shape and location (encleft-hit-hits, enc-right-hit-hits), where participants correctly identified the shape and location of stimuli during the test phase, successful encoding of shape but not location (enc-left-hit-misses, enc-right-hit-misses), where participants correctly identified the shape but not the location of stimuli during the test phase, and unsuccessful encoding of shape and location (enc-left-misses, enc-right-misses), where participants indicated old stimuli were new during the test phase (i.e., the latter stimuli were forgotten). Encoding-related activity associated with subsequent source memory was determined by contrasting enc-hithits $>$ enc-hit-misses, whereas encoding-related activity associated with subsequent item memory was determined by contrasting enc-hit-misses $>$ enc-misses. Encoding trials were assumed to be $2.5 \mathrm{sec}$ in duration (the length of stimulus presentation).

Retrieval events included successful retrieval of shapes and previous locations (old-left-hit-hits, old-right-hithits), successful retrieval of shapes but not location (old-left-hit-misses, old-right-hit-misses), unsuccessful retrieval of shapes (old-left-misses, old-right-misses), false memory of new shapes (new-left-false alarms, new-right-false alarms), correct rejection of new shapes (new-correct rejections), and failures to respond. 'No response' trials were assumed to be $2.5 \mathrm{sec}$ in duration, whereas other durations were measured from stimulus onset until the behavioral response.

Neural activation due to source memory was assessed by contrasting source memory (old-left-hit-hits and oldright-hit-hits) with item memory (old-left-hit-misses and old-right-hit-misses). In other words, activity associated with memory for both item and source information (oldhit-hits) was contrasted with activity associated with memory for item information alone (old-hit-misses). In 
conducting this contrast, we have assumed that the source memory judgment in this experiment involved both item recognition and memory for spatial location such that item memory effects can be directly subtracted out. It should be mentioned that we did not contrast old-hit-hits > correct rejections, as has sometimes been done, as this contrast does not subtract out item memory (and also suffers from an old vs. new stimulus type confound).

The neural activity associated with item memory was assessed by contrasting successful item memory without context (old-left-hit-misses and old-right-hit-misses) with unsuccessful item memory (old-left-misses and old-rightmisses). In other words, activity associated with correctly remembering the previously presented item, but not the source, was contrasted with activity associated with forgotten items.

A cluster extent threshold was enforced in order to correct for multiple comparisons (Ledberg, Åkerman, \& Roland, 1998; Forman et al., 1995; Poline \& Mazoyer, 1993; Roland, Levin, Kawashima, \& Åkerman, 1993). Specifically, an individual voxel statistical threshold of $p<.01$ was enforced, whereas a minimum cluster extent threshold of 137 resampled voxels was used to correct for multiple comparisons to $p<.01$. A Monte Carlo simulation with 10,000 iterations was run to determine the cluster extent necessary to correct for multiple comparisons. The Monte Carlo simulation modeled activity in each voxel using a normally distributed random number (mean of zero and unit variance) and type I error was assumed to be equal to the individual voxel threshold $p$ value in a volume defined by the functional acquisition dimensions. Spatial correlation was simulated by smoothing with a 6.2-mm full-width, half-maximum (FWHM) Gaussian, which was estimated using the $t$-statistic maps associated with contrasts of interest where the spatial autocorrelation of each slice was computed and the corresponding FWHM values were calculated (yielding an FWHM value of $6.17 \pm 0.21 \mathrm{~mm}$; similar procedures have been used previously to estimate fMRI spatial correlation, e.g., see Katanoda, Matsuda, \& Sugishita, 2002; Zarahn, Aguirre, \& D'Esposito, 1997). The probability of observing successively larger cluster sizes was computed based on the Monte Carlo cluster size distribution, and the cluster extent threshold was selected such that the probability of observing that or larger clusters was less than $p<.01$.

A conjunction analysis was conducted to determine whether there were any brain regions activated during both encoding and retrieval. Common source-memoryrelated activity was identified in those voxels that were active for both the encoding source memory contrast (enc-hit-hits $>$ enc-hit-misses) and the retrieval source memory contrast (old-hit-hits > old-hit-misses). Common item-memory-related activity was identified from the encoding item memory contrast (enc-hit-misses > enc-misses) and the retrieval item memory contrast (old- hit-misses $>$ old-misses). An individual voxel threshold of $p<.01$ was enforced for each contrast such that the joint probability of observing voxel activity in two such contrasts, as with these conjunctions, is equal to $p=.001$ (computed using Fisher's technique; Fisher, 1973; see also, Slotnick \& Schacter, 2004). For each conjunction, a voxel extent threshold of 46 contiguous resampled voxels was enforced, yielding correction for multiple comparisons to $p<.05$.

To assess memory retrieval effects in ROIs, the mean retrieval beta-weights were extracted from voxel coordinates $5 \mathrm{~mm}$ from the center of each ROI. MTL ROIs, within the hippocampus, parahippocampal cortex, and perirhinal cortex were examined. Activity in the hippocampus has previously been shown to increase in response to source memory (Dobbins et al., 2003; Cansino et al., 2002), allowing for an a priori prediction that the mean beta-weights in the hippocampus for old-hithits would be greater than the mean beta-weights for old-hit-misses. The perirhinal cortex was predicted to show a greater deactivation to old-hit-misses than oldmisses (i.e., an item-memory-related deactivation), oldhit-hits, and new-correct rejections during retrieval (as shown by Weis et al., 2004), where mean beta-weights were extracted at coordinates $(x=28, y=-16, z=-24)$ from Weis et al. (2004) as well as homologous coordinates in the contralateral hemisphere. MTL structures were delineated using anatomic descriptions (Bernasconi et al., 2003; Pruessner et al., 2002; Insausti et al., 1998).

Wheeler and Buckner (2004) showed that a region of the parietal cortex centered in the left intraparietal sulcus around BA 40/39 $(x=-39, y=-55, z=36)$ was active for both remember and know responses compared to correct rejections, whereas regions in the left lateral parietal $(x=-51, y=-51, z=38)$ and left posterior parietal cortex $(x=-43, y=-67, z=40)$ were only active for remember responses compared to correct rejections. Beta-weights were also extracted from these parietal regions as well as from homologous coordinates in the contralateral hemisphere.

Planned one-tailed paired $t$ tests were used to assess differences in beta-weights between event types based on the unidirectional nature of our predictions. In ROIs defined by the whole-brain analysis, one-tailed $t$ tests were used to assess differences between extracted beta-weights because the direction of differences between the betaweights was determined by the whole-brain (one-tailed) analysis. One-tailed $t$ tests were also used to assess differences in extracted beta-weights in the perirhinal cortex based on the previous finding by Weis et al. (2004) that old-hit-misses at the defined perirhinal ROI significantly deactivated the perirhinal cortex compared to old-hit-hits and old-misses. In the parietal cortex, one-tailed $t$ tests were again used to assess differences in extracted betaweights based on the previous findings of Wheeler and Buckner (2004), showing increased activation due to remember and know responses compared to correct 
rejections in the intraparietal sulcus and increased activation in the lateral, as well as posterior, parietal cortex for remember responses compared to correct rejections.

Event-related activity time courses were also extracted from spherical ROIs (radius $5 \mathrm{~mm}$ ) using custom software written in MATLAB. The activation time course (\% signal change as a function of time) associated with each trial of a given event type was first computed relative to stimulus onset and then these trials were averaged to produce the corresponding event-related time course (i.e., standard event-related averaging). Each time course was baseline corrected to produce an average of $0 \%$ signal change from 0 and $2 \mathrm{sec}$ before stimulus onset (for more details, see Slotnick \& Schacter, 2004). Previous work has suggested that time course analysis in regions outside the prefrontal cortex may not be as sensitive as beta-weight analysis. Also, time course analysis suffers from temporal autocorrelation, which may violate the data independence assumption (Slotnick, 2005). Therefore, statistics were not conducted on the event-related time course data but they are reported for illustrative purposes.

\section{RESULTS}

\section{Behavioral Results}

Participants' item memory, independent of spatial location accuracy, was $67.2 \pm 1.6 \%$ [computed from hit rate $\times$ $p($ old item $)+(1-$ false alarm rate $) \times p($ new item $)$, where hit rate $=70.2 \pm 2.6 \%$, false alarm rate $=38.9 \pm$ $3.3 \%]$. For items correctly identified as old, spatial location accuracy was $70.0 \pm 2.0 \%$ (Figure $2 \mathrm{~A}$ ). One-sample $t$ tests showed that item memory accuracy $[t(11)=$ $10.840, p<.001]$ and source memory accuracy $[t(11)=$ $10.135, p<.001]$ were both greater than chance performance $(50 \%)$ and were not significantly different from one another $[t(11)=1.629, p>.05]$. A repeatedmeasures analysis of variance revealed significant differences in reaction times across old-hit-hits, old-hit-misses, old-misses, and correct rejections $[F(3,33)=6.480, p<$ .001 ; Figure 2B]. Paired two-tailed $t$ tests revealed that old-hit-hits [2325.6 $\pm 44.24 \mathrm{msec}, t(11)=7.06, p<.001]$, old-misses [2399.61 $\pm 41.46 \mathrm{msec}, t(11)=2.32, p<.05$ ], and correct rejections [2354.24 $\pm 41.6 \mathrm{msec}, t(11)=$ $3.83, p<.01]$ were all significantly faster than old-hitmisses (2500.25 $\pm 41.43 \mathrm{msec})$, suggesting that item memory was more difficult than source memory, misses, and correct rejections. The finding that old-hit-misses were significantly longer than old-hit-hits is in agreement with previous research showing that retrieval with contextual information is faster than retrieval without contextual information (Montaldi et al., 2006; Woodruff, Johnson, Uncapher, \& Rugg, 2005; Weis et al., 2004; Wheeler \& Buckner, 2004; Cansino et al., 2002; Donaldson \& Rugg, 1998). There were a sufficient number of responses of each event type to conduct the fMRI analysis

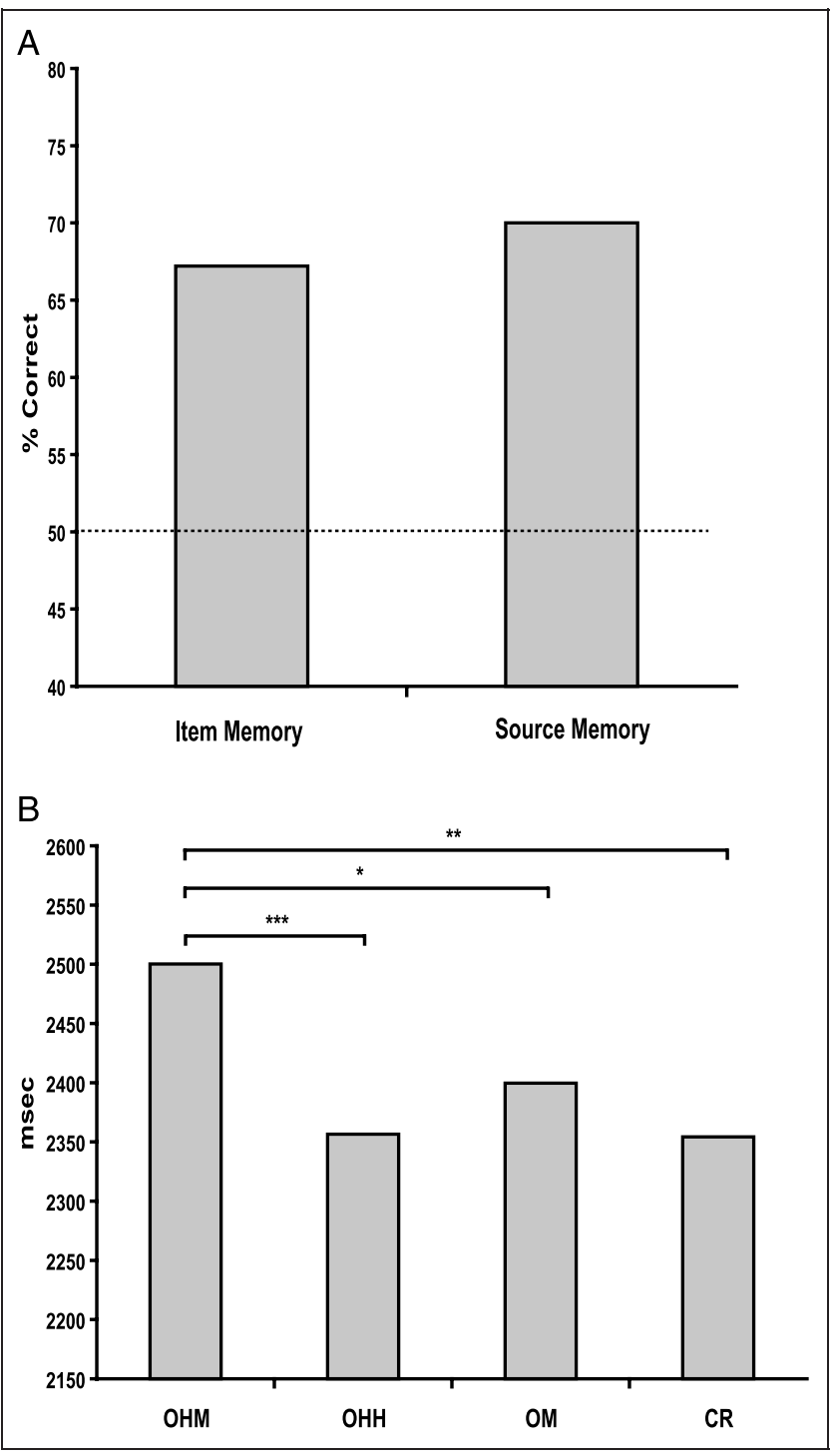

Figure 2. Behavioral results. (A) Mean percent correct for item memory and source memory. The dotted line represents chance performance. (B) Mean reaction time ( $\mathrm{msec}$ ) for old-hit-misses $(\mathrm{OHM})$, old-hit-hits $(\mathrm{OHH})$, old-misses $(\mathrm{OM})$, and correct rejections (CR). Asterisks denote a significant difference from old-hit-misses $(* p<.05, * * p<.01, * * * p<.001)$.

(old-hit-hits $91.42 \pm 5.25$, old-hit-misses $38.42 \pm 2.22$, oldmisses $55.75 \pm 5.24$, correct rejections $57.08 \pm 3.71$ ).

\section{fMRI Results}

Accurate source memory encoding (enc-hit-hits $>$ enchit-misses) was associated with activity in the left parahippocampal cortex, the intraparietal sulcus bilaterally, and the left fusiform gyrus (Figure 3; for a complete list of activations, see Table 1). Correct item memory encoding (enc-hit-misses > enc-misses) was associated with activity in the left inferior frontal sulcus and the left fusiform gyrus (Figure 3; for a complete list of activation, see Table 1). 
Figure 3. Whole-brain analysis results for both source memory and item memory. Green areas indicate activity during encoding. Red areas indicate activity during retrieval. Yellow areas indicate common encoding and retrieval activity. Activity shown is at the individual voxel threshold of $p<.01$, corrected for multiple comparisons to $p<.01$. Lighter blobs indicate activation further from the cortical surface, whereas darker blobs indicate activation near the cortical surface (LH = left hemisphere; $\mathrm{RH}=$ right hemisphere; $\mathrm{A}=$ anterior; $\mathrm{S}=$ superior).
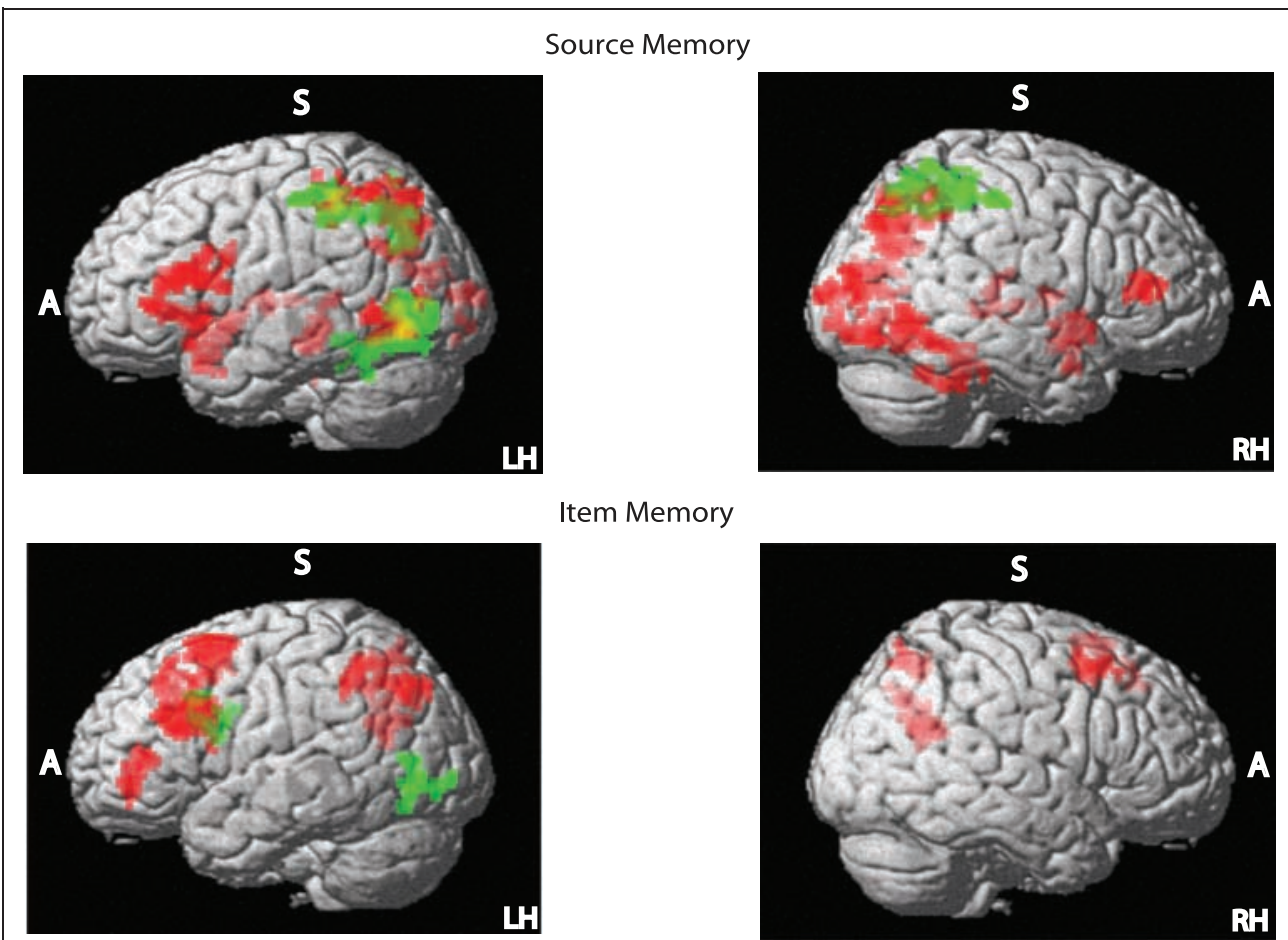

Table 1. Brain Regions Showing Memory Encoding Activation

\begin{tabular}{|c|c|c|c|c|}
\hline \multirow[b]{2}{*}{ Region } & \multicolumn{3}{|c|}{ Talairach Coordinates } & \multirow[b]{2}{*}{ Brodmann Area } \\
\hline & $x$ & $y$ & $z$ & \\
\hline \multicolumn{5}{|c|}{ Source Activations (enc-bit-bits > enc-bit-misses) } \\
\hline Left parahippocampal cortex & -24 & -41 & -9 & 36 \\
\hline Right postcentral gyrus & 56 & -21 & 46 & 2 \\
\hline Left postcentral gyrus & -51 & -24 & 50 & 2 \\
\hline Right supramarginal gyrus & 41 & -35 & 52 & 40 \\
\hline Right intraparietal sulcus & 33 & -42 & 52 & 40 \\
\hline Left intraparietal sulcus & -44 & -36 & 52 & 40 \\
\hline Right superior parietal lobule & 31 & -56 & 57 & 7 \\
\hline Left fusiform gyrus & -25 & -54 & -8 & $19 / 37$ \\
\hline Left middle temporal gyrus & -48 & -66 & 8 & $19 / 37$ \\
\hline Left inferior occipital gyrus & -46 & -76 & 0 & 19 \\
\hline Left middle occipital gyrus & -42 & -79 & 4 & 19 \\
\hline \multicolumn{5}{|c|}{ Item Activations (enc-hit-misses > enc-misses) } \\
\hline Left inferior frontal sulcus & -42 & 8 & 26 & $9 / 44$ \\
\hline Left inferior temporal gyrus & -42 & -73 & 1 & $19 / 37$ \\
\hline Left fusiform gyrus & -39 & -68 & -9 & $19 / 37$ \\
\hline Left inferior occipital gyrus & -38 & -81 & 0 & $18 / 19$ \\
\hline
\end{tabular}

Regions, Talairach coordinates and Brodmann areas refer to the center of each cluster of continuous voxels. 
Source memory retrieval (old-hit-hits $>$ old-hit-misses) was associated with activity in the bilateral inferior frontal gyrus, the intraparietal sulcus, the superior parietal lobule, the fusiform gyrus, and the putamen (Figure 3; for a complete list of activations, see Table 2). Source memory activations were also seen bilaterally in the hippocampus and in the left parahippocampal cortex (Figure 4A), suggesting greater hippocampus and parahippocampal cortex involvement in source memory compared to item memory. Item memory retrieval (old-hit-misses $>$ oldmisses) was associated with activity in the left inferior frontal gyrus, the superior frontal sulcus bilaterally, the precuneus, the posterior cingulate cortex, and the left intraparietal sulcus (Figure 3; Table 3). Interestingly, there were no significant activations associated with item memory retrieval at our individual voxel threshold of $p<$ .01 , corrected for multiple comparisons to $p<.01$ (see Methods) in the MTL, including the perirhinal cortex.

Our conjunction analysis showed that accurate encoding and retrieval of source memory were both associated with activity in the intraparietal sulcus bilaterally as well as the left inferior temporal gyrus and the left fusiform gyrus (Figure 3; for a complete list of activations, see Table 4). There were no statistically significant brain areas commonly activated for encoding and retrieval of item memory.

Mean beta-weights for old-hit-hits and old-hit-misses were extracted from the left and right hippocampus as well as the left parahippocampal cortex using the center of activations defined by the whole-brain analysis. A onetailed $t$ test revealed that the mean beta-weight associated with old-hit-hits was significantly greater than the mean beta-weight for old-hit-misses in the left hippocampus $[t(11)=2.828, p<.01]$ and right hippocampus $[t(11)=2.659, p<.05]$, showing greater involvement of the hippocampus during source memory retrieval compared to item memory, which is also supported by the event-related time course results (Figure 4A). In the left parahippocampal cortex, the mean beta-weight for oldhit-hits was also greater than the mean beta-weight for old-hit-misses $[t(11)=3.157, p<.01$; Figure 4A]. However, old-hit-hits and old-hit-misses had negative betaweights making it possible that old-hit-misses were more significantly deactivated than old-hit-hits, which was supported by the event-related time course results. Negative beta-weights are inherently difficult to interpret such that the role of the parahippocampal cortex in source memory retrieval is unclear.

In an effort to uncover item memory effects in the MTL, beta-weights were also extracted from the perirhinal cortex bilaterally based upon coordinates from Weis et al. (2004). There was a deactivation in the left perirhinal cortex in response to old-hit-misses that was significant when compared to old-hit-hits $[t(11)=2.739$, $p<.01]$ and old-misses $[t(11)=1.810, p<.05$; Figure $4 \mathrm{~B}]$, suggesting a role for the perirhinal cortex during retrieval of item memory.
In the parietal cortex, three different ROIs were defined using coordinates from Wheeler and Buckner (2004). A parietal cortex ROI near the intraparietal sulcus around BA 40/39 was found to be significantly activated bilaterally during retrieval of both old-hit-hits [left BA 40/39, $t(11)=$ $4.523, p<.001$; right $\mathrm{BA} 40 / 39, t(11)=2.490, p<.05]$ and old-hit-misses [left BA 40/39, $t(11)=4.837, p<.001$; right $\mathrm{BA} 40 / 39, t(11)=2.967, p<.05]$ compared to correct rejections (Figure 5), consistent with Wheeler and Buckner's finding of an increase in activation in response to both remember and know responses compared to correct rejections in this region. An $\mathrm{ROI}$ in the lateral parietal cortex showed a strikingly different pattern where correct rejections showed a significant deactivation bilaterally compared to both old-hit-hits [left, $t(11)=3.892, p<.01$; right, $t(11)=2.282, p<.05$ ] and old-hit-misses [left, $t(11)=$ $2.126, p<.05$; right, $t(11)=2.246, p<.05]$. An ROI in the left posterior parietal cortex showed the same pattern where correct rejections showed a significant deactivation compared to both old-hit-hits $[t(11)=3.325, p<.01]$ and old-hit-misses $[t(11)=2.817, p<.01]$. These latter findings of greater deactivation in response to correct rejections compared to old-hit-hits and old-hit-misses in the lateral and posterior parietal cortex are in contrast to Wheeler and Buckner, who observed decreased activity in these regions for both correct rejections and know responses. The event-related time course results in the parietal cortex generally followed the same pattern of activation as seen in the beta-weight analysis with one exception. In the lateral parietal cortex bilaterally, the event-related time course results suggest that there may be no differential activity in this region. However, this was not entirely surprising because time course analysis in regions outside the prefrontal cortex does not appear to be as sensitive as beta-weight analysis (cf., Slotnick, 2005).

Item memory retrieval was also found to activate the left inferior frontal gyrus, near BA 10/46, in the wholebrain analysis. Mean beta-weights and event-related time courses were also extracted from this region bilaterally. One-tailed $t$ test revealed that the mean beta-weight for old-hit-misses was significantly greater than the mean beta-weight for old-misses in this region $[t(11)=3.905$, $p<.01$; Figure 6], which was supported by the eventrelated time course results. By comparison, there were no item-memory-related differences in the right inferior frontal gyrus $[t(11)=0.864, p>.05$; Figure 6].

\section{DISCUSSION}

The main finding from the present study suggests that the hippocampus is activated more strongly when retrieving contextual information than when retrieving item information. When item-memory-related activity was directly subtracted out from source memory activation, the hippocampus was activated bilaterally. Examination of the mean beta-weights for each event type revealed greater hippocampal activation during old-hit- 
Table 2. Brain Regions Showing Source Memory Activation (Old-Hit-Hits > Old-Hit-Misses)

\begin{tabular}{|c|c|c|c|c|}
\hline \multirow[b]{2}{*}{ Region } & \multicolumn{3}{|c|}{ Talairach Coordinates } & \multirow[b]{2}{*}{ Brodmann's Area } \\
\hline & $x$ & $y$ & $z$ & \\
\hline Right amygdala & 27 & 2 & -20 & - \\
\hline Left hippocampus & -16 & -31 & 0 & - \\
\hline Right hippocampus & 19 & -31 & 0 & - \\
\hline Left parahippocampal cortex & -29 & -33 & -11 & 36 \\
\hline Right inferior frontal gyrus & 44 & 33 & 9 & $45 / 46$ \\
\hline Left inferior frontal gyrus & -47 & 24 & 14 & $44 / 45 / 46$ \\
\hline Left inferior frontal gyrus & -46 & 6 & 21 & 44 \\
\hline Left insula & -35 & 21 & -2 & 13 \\
\hline Right insula & 37 & 12 & -7 & 13 \\
\hline Left orbital gyrus & -28 & 14 & -19 & 11 \\
\hline Left intraparietal sulcus & -34 & -50 & 53 & $7 / 19 / 39 / 40$ \\
\hline Right intraparietal sulcus & 28 & -50 & 49 & $7 / 39 / 40$ \\
\hline Left superior parietal lobule & -23 & -63 & 45 & 7 \\
\hline Right superior parietal lobule & 29 & -65 & 44 & 7 \\
\hline Left precuneus & -11 & -66 & 49 & 7 \\
\hline Right precuneus & 14 & -63 & 30 & $7 / 31$ \\
\hline Left temporopolar cortex & -28 & 4 & -24 & 38 \\
\hline Left inferior temporal sulcus & -44 & -67 & -2 & $19 / 21 / 37$ \\
\hline Left inferior temporal sulcus & -48 & -54 & 2 & $19 / 21 / 37$ \\
\hline Right inferior temporal gyrus & 51 & -65 & 0 & $21 / 37$ \\
\hline Right middle temporal gyrus & 48 & -60 & -1 & 37 \\
\hline Right fusiform gyrus & 47 & -51 & -10 & 37 \\
\hline Right fusiform gyrus & 30 & -76 & -6 & 19 \\
\hline Left fusiform gyrus & -42 & -57 & -8 & 37 \\
\hline Right lingual gyrus & 19 & -74 & 1 & 18 \\
\hline Right striate cortex & 19 & -71 & 10 & 17 \\
\hline Right cuneus & 12 & -74 & 20 & 18 \\
\hline Left middle occipital gyrus & -40 & -63 & 5 & 37 \\
\hline Right putamen & 27 & 5 & -3 & - \\
\hline Left putamen & -25 & 6 & -5 & - \\
\hline Left globus pallidus & -10 & 3 & -5 & - \\
\hline Right thalamus (anterior nucleus) & 7 & -7 & 5 & - \\
\hline Left thalamus (dorsomedial nucleus) & -5 & -11 & 5 & - \\
\hline Right thalamus (dorsomedial nucleus) & 6 & -7 & 6 & - \\
\hline Right thalamus (pulvinar) & 14 & -28 & 9 & - \\
\hline Left thalamus (pulvinar) & -4 & -28 & 0 & - \\
\hline Right cerebellum & 35 & -41 & -25 & - \\
\hline
\end{tabular}

Regions, Talairach and Tournoux (1988) coordinates, and Brodmann's areas refer to the center of each cluster of continuous voxels. 


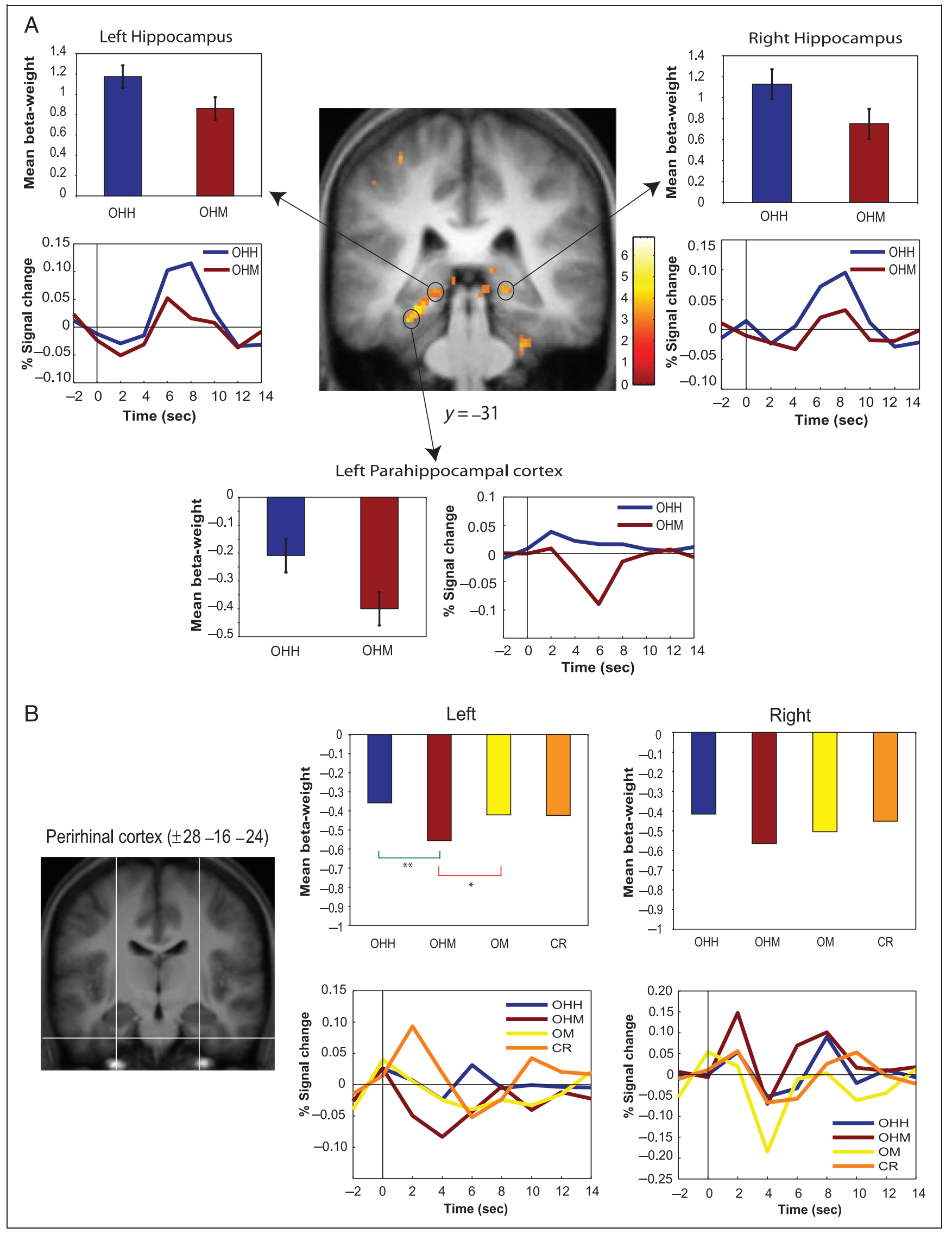


Table 3. Brain Regions Showing Item Memory Activation (Old-Hit-Misses > Old-Misses)

\begin{tabular}{|c|c|c|c|c|}
\hline \multirow[b]{2}{*}{ Region } & \multicolumn{3}{|c|}{ Talairach Coordinates } & \multirow[b]{2}{*}{ Brodmann's Area } \\
\hline & $x$ & $y$ & $z$ & \\
\hline Left inferior frontal gyrus & -40 & 42 & -5 & $10 / 46$ \\
\hline Left inferior frontal sulcus & -42 & 22 & 20 & $9 / 46$ \\
\hline Left superior frontal sulcus & -24 & 17 & 52 & 6/8/9 \\
\hline Right superior frontal sulcus & 34 & 13 & 47 & 6 \\
\hline Left intraparietal sulcus & -40 & -45 & 47 & $7 / 40$ \\
\hline Left angular gyrus & -40 & -66 & 39 & 39 \\
\hline Left precuneus & -1 & -62 & 37 & 7 \\
\hline Right precuneus & 1 & -58 & 53 & 7 \\
\hline Left posterior cingulate & -13 & -59 & 24 & $23 / 30$ \\
\hline Right posterior cingulate & 13 & -58 & 26 & $23 / 30$ \\
\hline
\end{tabular}

Regions, Talairach and Tournoux (1988) coordinates, and Brodmann's areas refer to the center of each cluster of continuous voxels.

hits than old-hit-misses. It is important to note that this finding does not imply that item information does not rely on the hippocampus, simply that the hippocampus is more strongly activated during retrieval of contextual information. The hippocampus has long been associated with spatial memory, and recent evidence has led to the hypothesis that the hippocampus plays a key role in binding information together during all forms of episodic memory, including spatial and nonspatial information (Manns \& Eichenbaum, 2006; Eichenbaum, 2000). Studies in humans have shown strong hippocampal activation when encoding relational information (Davachi \& Wagner, 2002) and when retrieving associations (Small et al., 2001; Yonelinas, Hopfinger, Buonocore, Kroll, \& Baynes, 2001), and focal lesion evidence has also revealed that damage limited to the hippocampus impairs associative memory more than item memory (Holdstock, Mayes, Gong, Roberts, \& Kapur, 2005; Mayes et al., 2004). It is probable that the increased hippocampal activation seen in this study during spatial source memory is due to retrieval of contextual (spatial location) information associated with an item during the study phase.

The results of our analyses also suggest that the parahippocampal cortex is more activated during contextual memory than during item memory, suggesting a role for this region during retrieval of spatial contextual information. However, this result is more difficult to interpret. When mean beta-weights were extracted from the left parahippocampal cortex, the "activity" in this region was associated with item- and source-memoryrelated deactivations (see Figure 4A). Previous work has shown a similar pattern where parahippocampal cortical deactivation was seen during item and context memory (Burgess et al., 2001). Parahippocampal deactivation has also been seen during know responses compared to new responses (Woodruff et al., 2005). However, other studies have shown significant increases in parahippocampal cortex activation during retrieval of spatial location (Ekstrom et al., 2003) as well as spatial and nonspatial contextual information (Aminoff, Gronau, \& Bar, 2006; Bar \& Aminoff, 2003). It appears then that the nature of the stimuli and task demands determine whether the parahippocampal cortex is activated or deactivated during retrieval. Further work will be necessary to determine the functional role of activations versus deactivations in the parahippocampal cortex.

The results from our subsequent memory analysis revealed that the parahippocampal cortex was associated with successful encoding of spatial source information, which is consistent with previous research showing subsequent source memory effects in this region (Davachi et al., 2003; Ranganath et al., 2003). Unlike Davachi et al. (2003) and Ranganath et al. (2003), we found no subsequent source memory effects in the hippocampus nor were there any subsequent item memory effects in the perirhinal cortex. The lack of subsequent source memory effects

Figure 4. Medial-temporal lobe activations (left hemisphere toward left). (A) Hippocampal and parahippocampal cortex activations associated with the source memory contrast (old-hit-hits > old-hit-misses). Mean beta-weights and event-related time courses defined from our whole-brain analysis were extracted for old-hit-hits (OHH, blue bars and lines) and old-hit-misses (OHM, red bars and lines). Error bars reflect within-subject standard errors of the mean. (B) Mean beta-weights and event-related time courses extracted bilaterally from the perirhinal cortex using coordinates defined from Weis et al. (2004) for old-hit-hits (OHH, blue bars and lines), old-hit-misses (OHM, red bars and lines), old-misses (OM, yellow bars and lines), and correct rejections (CR, orange bars and lines). Asterisks denote a significant difference from old-hit-misses $(* p<.05$, ** $p<.01)$. 
Table 4. Brain Regions Showing Both Source Memory Encoding and Source Memory Retrieval Activations (Enc-Hit-Hits $>$ Enc-HitMisses $\cap$ Old-Hit-Hits $>$ Old-Hit-Misses)

\begin{tabular}{|c|c|c|c|c|}
\hline \multirow[b]{2}{*}{ Region } & \multicolumn{3}{|c|}{ Talairach Coordinates } & \multirow[b]{2}{*}{ Brodmann's Area } \\
\hline & $x$ & $y$ & $z$ & \\
\hline Left inferior temporal gyrus & -43 & -67 & -2 & 37 \\
\hline Left fusiform gyrus & -42 & -58 & -8 & 37 \\
\hline Left intraparietal sulcus & -22 & -59 & 46 & 7 \\
\hline Right intraparietal sulcus & 29 & -56 & 52 & 7 \\
\hline
\end{tabular}

Regions, Talairach and Tournoux (1988) coordinates, and Brodmann's areas refer to the center of each cluster of continuous voxels.

in the hippocampus is not without precedent (Gold et al., 2006; Cansino et al., 2002). In the current study, it is likely that a lack of power contributed to the null subsequent memory findings in the hippocampus and in the perirhinal cortex because we designed the imaging protocol to investigate item and source memory effects at retrieval.

An ROI analysis using coordinates from Weis et al. (2004) revealed a deactivation in the left perirhinal cortex during item memory compared to source memory, forgotten items, and correct rejections, consistent with the results of that previous study. However, it should be pointed out that our whole-brain analysis did not reveal item memory retrieval related activity in the perirhinal cortex. Unlike the parahippocampal cortex deactivations described above, there is a wealth of information concerning deactivations in the perirhinal cortex. Activity in the perirhinal cortex in rats, measured using the immediate early gene c-fos, decreases upon subsequent presentation of a stimulus (Wan, Aggleton, \& Brown, 1999), suggesting that this region signals familiarity (Brown \& Aggleton, 2001). Electrophysiological recordings in monkeys (Xiang \& Brown, 1998; Fahy et al., 1993) and humans (Halgren et al., 2006) show that neurons in the perirhinal cortex decrease their firing rate upon subsequent exposure to a stimulus. Human fMRI studies have also shown that the perirhinal cortex is significantly deactivated during item memory (Weis et al., 2004) and may signal stimulus familiarity (Montaldi et al., 2006; Henson et al., 2003). Combined, the results from rats, monkeys, and humans all suggest that the perirhinal cortex deactivates with stimulus familiarity, which makes it likely that the deactivation seen in the perirhinal cortex in the current study signals familiarity during item memory.

Item memory activation during retrieval was also found in the left prefrontal cortex, near BA 10/46, which is in contrast with previous results. Item memory has been preferentially associated with the right prefrontal cortex, whereas source memory has been preferentially associated with the left prefrontal cortex (see Slotnick, Moo, Segal, \& Hart, 2003 for review). Although our whole-brain analysis did reveal item memory activation in the right prefrontal cortex, the left prefrontal hemispheric asymmetry is notable (see Figures 3 and 6).

Our results revealed increased hippocampal activation during source memory. One line of thought suggests that the hippocampus is activated during source memory because this cognitive process is more difficult than item memory. However, the results from the current study do not support this hypothesis. The behavioral results revealed that item and source memory accuracies did not significantly differ. Furthermore, source memory reaction times were significantly faster than item memory reaction times, a finding consistent with prior source memory experiments (Weis et al., 2004; Cansino et al., 2002; Donaldson \& Rugg, 1998). Together, the accuracy and reaction time data suggest that source memory difficulty is at least equivalent, if not easier, than item memory in the current study. Because our results show greater hippocampal activation during source memory, as compared to item memory, it is unlikely that the hippocampus is simply responding to differences in difficulty between item and source memory.

The results from the current study also revealed source memory activation in the putamen bilaterally (Table 2, Figure 7). In light of recent findings showing that activation in the putamen correlates with spatial navigation ability (Epstein, Higgins, \& Thompson-Schill, 2005), it is not surprising that we found activation of the putamen during spatial source memory retrieval.

Three different ROIs in the parietal cortex were taken from Wheeler and Buckner (2004) to determine whether the same functional dissociation for remember and know responses existed in these regions during item and source memory. Consistent with previous findings, the intraparietal sulcus ROIs near BA 40/39 were associ-

Figure 5. Mean beta-weights and event-related time courses extracted from the parietal cortex ROIs (shown at center, left hemisphere toward top, anterior toward right) using coordinates defined from Wheeler and Buckner (2004) for OHH (black bars and solid lines), OHM (dark gray bars and dotted lines), and CR (light gray bars and dashed lines). Asterisks denote a significant difference from new-correct rejections $(* p<.05, * * p<.01)$. 

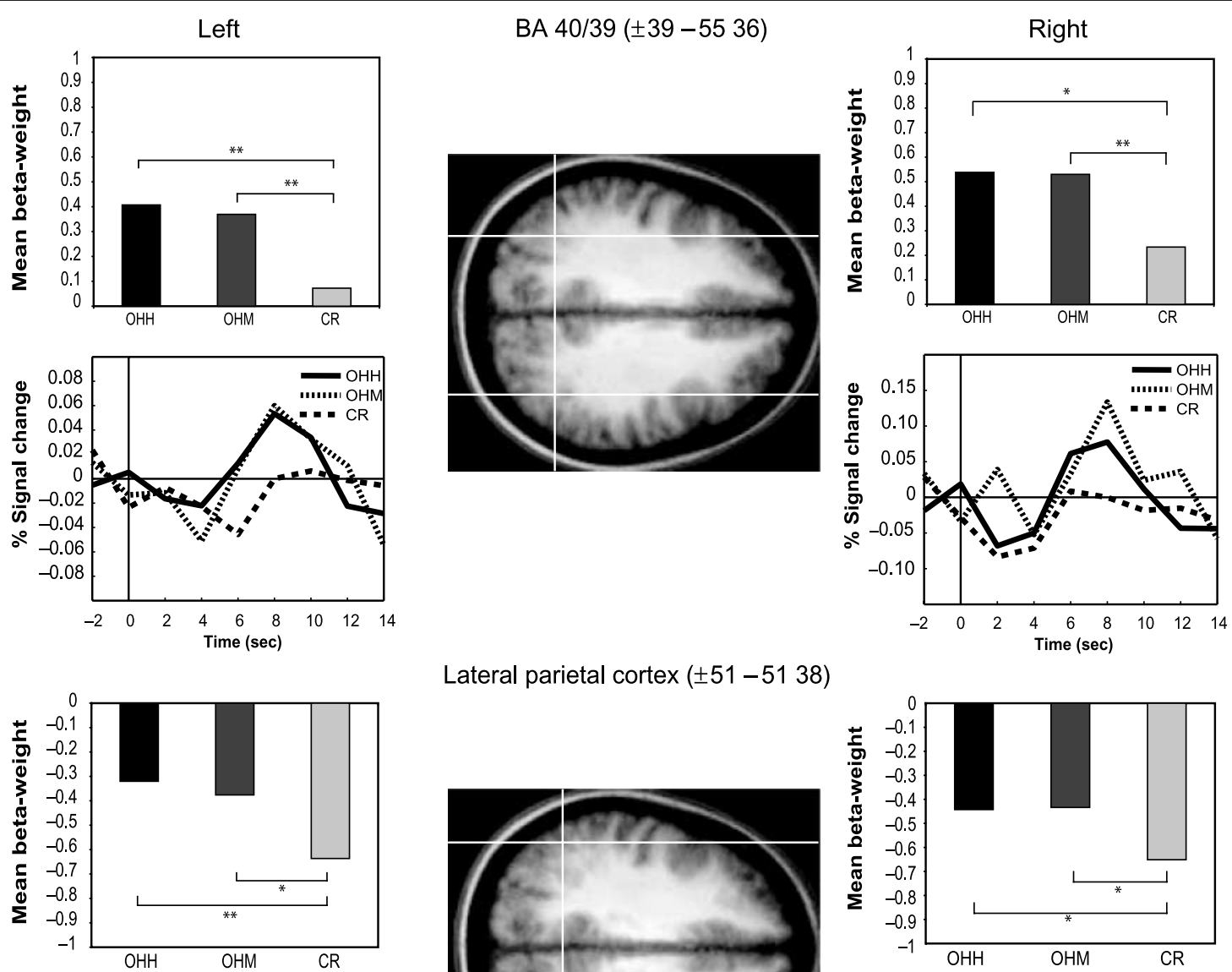

Lateral parietal cortex $( \pm 51-5138)$
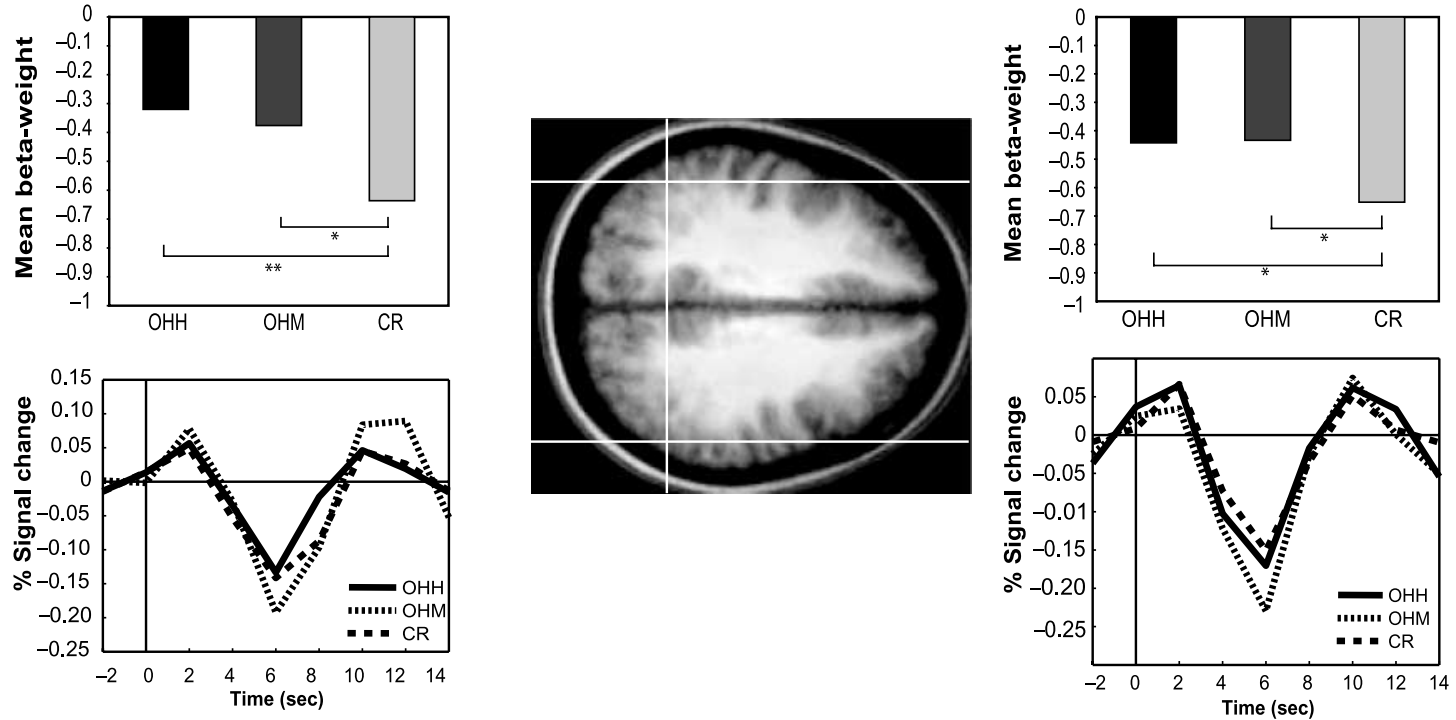

Posterior parietal cortex $( \pm 43-6740)$
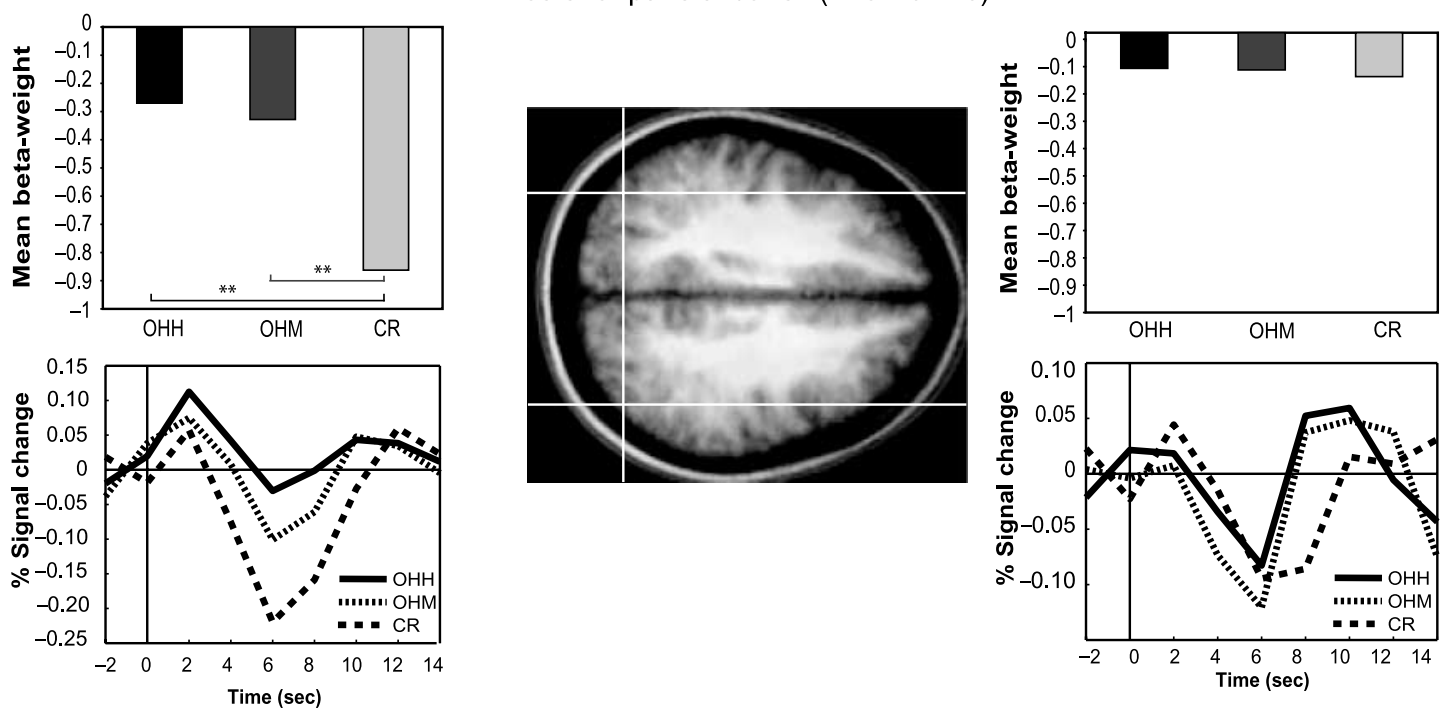


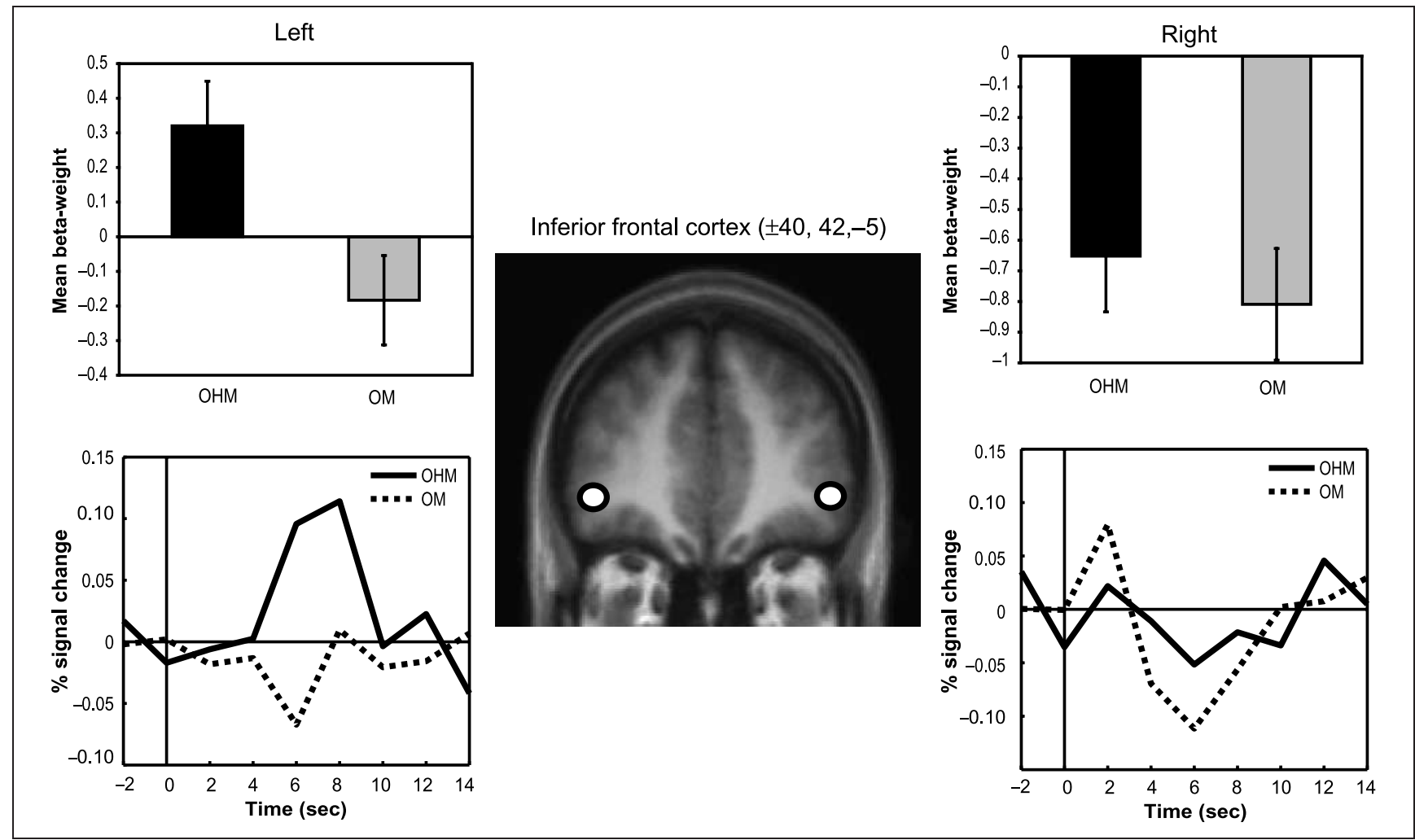

Figure 6. Mean beta-weights and event-related time courses extracted from the inferior frontal cortex (left hemisphere toward left, superior toward top) using coordinates defined from our whole-brain analysis for OHM (dark gray bars and solid lines) and OM (light gray bars and dashed lines).

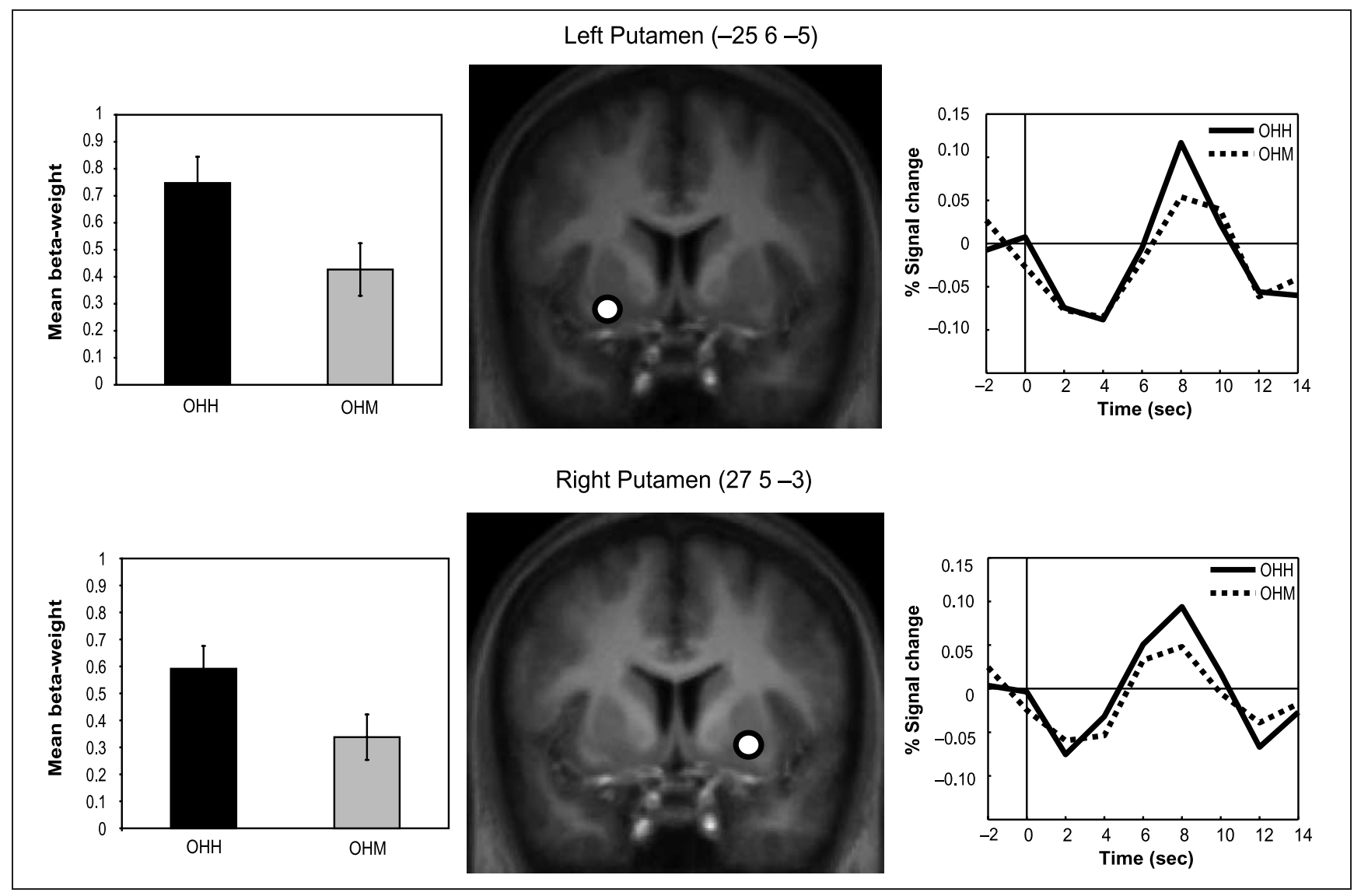

Figure 7. Mean beta-weights and event-related time courses extracted from the putamen (left hemisphere toward left, superior toward top) using coordinates defined from our whole-brain analysis for OHH (dark gray bars and solid lines) and OHM (light gray bars and dashed lines). 
ated with greater activity for both old-hit-hits and oldhit-misses compared to correct rejections, suggesting that this region responds to old stimuli regardless of contextual information associated with an item (Wheeler \& Buckner, 2003, 2004; Konishi et al., 2000). However, there was a region of the intraparietal sulcus, near BA 7, which was active during successful encoding of spatial source information in the subsequent memory analysis. Moreover, results from the conjunction analysis revealed that this region was also active during the retrieval of spatial source information, suggesting that a more superior part of the intraparietal sulcus in BA 7 is important for both the encoding and retrieval of spatial source information.

In the lateral parietal cortex ROIs bilaterally and the left posterior parietal cortex ROI, it was found that correct rejections showed greater deactivation than both old-hit-hits and old-hit-misses, suggesting that these regions are more strongly deactivated to correctly rejected new stimuli regardless of contextual retrieval success. In comparison, Wheeler and Buckner (2004) showed that these lateral and posterior parietal cortex ROIs were significantly deactivated during correct rejections and know responses compared to remember responses, suggesting a functional segregation in the parietal cortex for remember and know responses. Our findings suggest that the same functional segregation that may exist for remember and know responses does not exist for item and source memory. It is possible that this difference is due to the subjective nature of the remember/know task versus the objective nature of the item and source memory task.

Of relevance to our primary aim, the results from the present study support the hypothesis that the hippocampus is more strongly activated during contextual memory than during item memory. The parahippocampal cortex was also associated with contextual memory, but this was driven by a deactivation, which is difficult to interpret. In the perirhinal cortex, item memory was associated with the greatest deactivation, as compared to source memory, forgotten items, and correct rejections, in line with theories suggesting that a decrease in perirhinal cortex activity signals familiarity. Considered together, these results support the hypothesis that distinct subregions of the MTL are preferentially associated with item memory and memory for context.

Reprint requests should be sent to Robert S. Ross, Conte Center for Memory and Brain, Department of Psychology, Boston University, 2 Cummington St, Boston, MA 02215-2425, or via e-mail: bross@bu.edu.

\section{REFERENCES}

Aminoff, E., Gronau, N., \& Bar, M. (2006). The parahippocampal cortex mediates spatial and nonspatial associations. Cerebral Cortex, 17, 1493-1503.

Bar, M., \& Aminoff, E. (2003). Cortical analysis of visual context. Neuron, 38, 347-358.
Bernasconi, N., Bernasconi, A., Caramanos, Z., Antel, S. B., Andermann, F., \& Arnold, D. L. (2003). Mesial temporal damage in temporal lobe epilepsy: A volumetric MRI study of the hippocampus, amygdala and parahippocampal region. Brain, 126, 462-469.

Brown, M. W., \& Aggleton, J. P. (2001). Recognition memory: What are the roles of the perirhinal cortex and hippocampus. Nature Reviews: Neuroscience, 2, 51-61.

Burgess, N., Maguire, E. A., Spiers, H. J., \& O'Keefe, J. (2001). A temporoparietal and prefrontal network for retrieving the spatial context of lifelike events. Neuroimage, 14, 439-453.

Cansino, S., Maquet, P., Dolan, R. J., \& Rugg, M. D. (2002). Brain activity underlying encoding and retrieval of source memory. Cerebral Cortex, 12, 1048-1056.

Davachi, L., Mitchell, J. P., \& Wagner, A. D. (2003). Multiple routes to memory: Distinct medial temporal lobe processes build item and source memories. Proceedings of the National Academy of Sciences, U.S.A., 100, 2157-2162.

Davachi, L., \& Wagner, A. D. (2002). Hippocampal contributions to episodic encoding: Insights from relational and item-based learning. Journal of Neurophysiology, 88, 982-990.

Dobbins, I. G., Rice, H. J., Wagner, A. D., \& Schacter, D. L. (2003). Memory orientation and success: Separable neurocognitive components underlying episodic recognition. Neuropsychologia, 41, 318-333.

Dobbins, I. G., Simons, J. S., \& Schacter, D. L. (2004). fMRI evidence for separable and lateralized prefrontal memory monitoring processes. Journal of Cognitive Neuroscience, 16, 908-920.

Donaldson, D. I., \& Rugg, M. D. (1998). Recognition memory for new associations: Electrophysiological evidence for the role of recollection. Neuropsychologia, 36, 377-395.

Eichenbaum, H. (2000). A cortical-hippocampal system for declarative memory. Nature Reviews: Neuroscience, 1, 41-50.

Ekstrom, A. D., Kahana, M. J., Caplan, J. B., Fields, T. A., Isham, E. A., Newman, E. L., et al. (2003). Cellular networks underlying human spatial navigation. Nature, 425, 184-187.

Epstein, R. A., Higgins, J. S., \& Thompson-Schill, S. L. (2005). Learning places from views: Variation in scene processing as a function of experience and navigational ability. Journal of Cognitive Neuroscience, 17, 73-83.

Fahy, F. L., Riches, I. P., \& Brown, M. W. (1993). Neuronal activity related to visual recognition memory: Long-term memory and the encoding of recency and familiarity information in the primate anterior and medial inferior temporal and rhinal cortex. Experimental Brain Research, 96, 457-472.

Fisher, R. A. (1973). Statistical methods for research workers (14th ed.). New York: Hafner.

Forman, S. D., Cohen, J. D., Fitzgerald, M., Eddy, W. F., Mintun, M. A., \& Noll, D. C. (1995). Improved assessment of significant activation in functional magnetic resonance imaging (fMRI): Use of a cluster-size threshold. Magnetic Resonance in Medicine, 33, 636-647.

Gold, J. J., Smith, C. N., Bayley, P. J., Shrager, Y., Brewer, J. B., Stark, C. E. L., et al. (2006). Item memory, source memory, and the medial temporal lobe: Concordant findings from fMRI and memory-impaired patients. Proceedings of the National Academy of Sciences, U.S.A., 103, 9351-9356.

Halgren, E., Wang, C., Schomer, D. L., Knake, S., Marinkovic, K., Wu, J., et al. (2006). Processing stages underlying word recognition in the anteroventral temporal lobe.

Neuroimage, 30, 1401-1413. 
Henson, R. N. A., Cansino, S., Herron, J. E., Robb, W. G. K., \& Rugg, M. D. (2003). A familiarity signal in human anterior medial temporal cortex? Hippocampus, 13, 301-304.

Holdstock, J. S. (2005). The role of the human medial temporal lobe in object recognition and object discrimination. Quarterly Journal of Experimental Psychology, 58B, 326-339.

Holdstock, J. S., Mayes, A. R., Gong, Q. Y., Roberts, N., \& Kapur, N. (2005). Item recognition is less impaired than recall and associative recognition in a patient with selective hippocampal damage. Hippocampus, 15, 203-215.

Insausti, R., Juottonen, K., Soininen, H., Insausti, A. M., Partanen, K., Vainio, P., et al. (1998). MR volumetric analysis of the human entorhinal, perirhinal, and temporopolar cortices. AJNR. American Journal of Neuroradiology, 19, 659-671.

Katanoda, K., Matsuda, Y., \& Sugishita, M. (2002). A spatio-temporal regression model for the analysis of functional MRI data. Neuroimage, 17, 1415-1428.

Konishi, S., Wheeler, M. E., Donaldson, D. I., \& Buckner, R. L. (2000). Neural correlates of episodic retrieval success. Neuroimage, 12, 276-286.

Ledberg, A., Akerman, S., \& Roland, P. E. (1998). Estimation of the probabilities of $3 \mathrm{D}$ clusters in functional brain images. Neuroimage, 8, 113-128.

Manns, J. R., \& Eichenbaum, H. (2006). Evolution of declarative memory. Hippocampus, 16, 795-808.

Manns, J. R., Hopkins, R. O., Reed, J. M., Kitchener, E. G., \& Squire, L. R. (2003). Recognition memory and the human hippocampus. Neuron, 37, 171-180.

Mayes, A. R., Holdstock, J. S., Isaac, C. L., Montaldi, D., Grigor, J., Gummer, A., et al. (2004). Associative recognition in a patient with selective hippocampal lesions and relatively normal item recognition. Hippocampus, 14, 763-784.

Montaldi, D., Spencer, T. J., Roberts, N., \& Mayes, A. R. (2006). The neural system that mediates familiarity memory. Hippocampus, 16, 504-520.

Poline, J. B., \& Mazoyer, B. M. (1993). Analysis of individual positron emission tomography activation maps by detection of high signal-to-noise-ratio pixel clusters. Journal of Cerebral Blood Flow and Metabolism, 13, 425-437.

Pruessner, J. C., Köhler, S., Crane, J., Pruessner, M., Lord, C., Byrne, A., et al. (2002). Volumetry of temporopolar, perirhinal, entorhinal and parahippocampal cortex from high-resolution MR images: Considering the variability of the collateral sulcus. Cerebral Cortex, 12, 1342-1353.

Ranganath, C., Yonelinas, A. P., Cohen, M. X., Dy, C. J., Tom, S. M., \& D'Esposito, M. (2003). Dissociable correlates of recollection and familiarity within the medial temporal lobes. Neuropsychologia, 42, 2-13.

Roland, P. E., Levin, B., Kawashima, R., \& Åkerman, S. (1993). Three-dimensional analysis of clustered voxels in 15-O-butanol brain activation images. Human Brain Mapping, 1, 3-19.

Slotnick, S. D. (2005). Spatial working memory specific activity in dorsal prefrontal cortex? Disparate answers from fMRI beta-weight and timecourse analysis. Cognitive Neuropsychology, 22, 905-920.

Slotnick, S. D., Moo, L. R., Segal, J. B., \& Hart, J., Jr. (2003). Distinct prefrontal cortex activity associated with item memory and source memory for visual shapes. Cognitive Brain Research, 17, 75-82.

Slotnick, S. D., \& Schacter, D. L. (2004). A sensory signature that distinguishes true from false memories. Nature Neuroscience, 7, 664-672.

Small, S. A., Nava, A. S., Perera, G. M., DeLaPaz, R., Mayeux, R., \& Stern, Y. (2001). Circuit mechanisms underlying memory encoding and retrieval in the long axis of the hippocampal formation. Nature Neuroscience, 4, 442-449.

Squire, L. R., Stark, C. E. L., \& Clark, R. E. (2004). The medial temporal lobe. Annual Review of Neuroscience, 27, 279-306.

Stark, C. E. L., \& Squire, L. R. (2001). Simple and associative recognition memory in the hippocampal region. Learning and Memory, 8, 190-197.

Talairach, J., \& Tournoux, P. (1988). Co-planar stereotaxic atlas of the buman brain. New York: Thieme.

Wan, H., Aggleton, J. P., \& Brown, M. W. (1999). Different contributions of the hippocampus and perirhinal cortex to recognition memory. Journal of Neuroscience, 19, $1142-1148$

Weis, S., Specht, K., Klaver, P., Tendolkar, I., Willmes, K., Ruhlmann, J., et al. (2004). Process dissociation between contextual retrieval and item recognition. NeuroReport, 15, 2729-2733.

Wheeler, M. E., \& Buckner, R. L. (2003). Functional dissociation among components of remembering: Control, perceived oldness, and content. Journal of Neuroscience, 23, 3869-3880.

Wheeler, M. E., \& Buckner, R. L. (2004). Functional-anatomic correlates of remembering and knowing. Neuroimage, 21, 1337-1349.

Woodruff, C. C., Johnson, J. D., Uncapher, M. R., \& Rugg, M. D. (2005). Content-specificity of the neural correlates of recollection. Neuropsychologia, 43, 1022-1032.

Xiang, J. Z., \& Brown, M. W. (1998). Differential neuronal encoding of novelty, familiarity and recency in regions of the anterior temporal lobe. Neuropharmacology, 37, 657-676.

Yonelinas, A. P., Hopfinger, J. B., Buonocore, M. H., Kroll, N. E. A., \& Baynes, K. (2001). Hippocampal, parahippocampal and occipital-temporal contributions to associative and item recognition memory: An fMRI study. NeuroReport, 12, 359-363.

Yonelinas, A. P., Kroll, N. E. A., Quamme, J. R., Lazzara, M. M., Sauve, M.-J., Widaman, K. F., et al. (2002). Effects of extensive temporal lobe damage or mild hypoxia on recollection and familiarity. Nature Neuroscience, 5 , 1236-1241.

Zarahn, E., Aguirre, G. K., \& D’Esposito, M. (1997). Empirical analysis of BOLD fMRI statistics: I. Spatially unsmoothed data collected under null-hypothesis conditions. Neuroimage, 5, 179-197. 